POS $\quad$ PROCEEDINGS

\title{
Type Iln supernovae as sources of high energy neutrinos
}

\author{
V.N.Zirakashvili* \\ Pushkov Institute of Terrestrial Magnetism, Ionosphere and Radiowave Propagation, 142190, \\ Troitsk, Moscow, Russia \\ E-mail: zirakeizmiran.ru
}

\section{V.S.Ptuskin}

Pushkov Institute of Terrestrial Magnetism, Ionosphere and Radiowave Propagation, 142190,

Troitsk, Moscow, Russia

E-mail: vptuskin@izmiran.ru

It is shown that high-energy astrophysical neutrinos observed in the IceCube experiment can be produced by protons accelerated in extragalactic Type IIn supernova remnants by shocks propagating in the dense circumstellar medium. The nonlinear diffusive shock acceleration model is used for description of particle acceleration.

The 34th International Cosmic Ray Conference,

30 July- 6 August, 2015

The Hague, The Netherlands

${ }^{*}$ Speaker. 


\section{Introduction}

The detection of very high energy astrophysical neutrinos in the IceCube experiment $[1,2]$ opens up a new possibility for investigation of particle acceleration processes in the Universe. The neutrino production in cosmos is possible via the $p p$ and $p \gamma$ interactions and the decay chains $\pi^{+} \rightarrow \mu^{+} v_{\mu}, \mu^{+} \rightarrow e^{+} v_{e} \overline{v_{\mu}}$. The observed astrophysical flux emerges from under more steep air show spectrum at about $50 \mathrm{TeV}$ and has a cutoff at $2 \mathrm{PeV}$. The neutrino typically carries small part of the primary proton energy, $E_{v} \approx 0.05 E_{p}$, so the protons with energies up to $E_{\max } \sim 10^{17} \mathrm{eV}$ are required to explain the observations (this energy is $\sim 10^{17} \mathrm{eV} /$ nucleon in the case of neutrino production by nuclei). Assuming an $E^{-2}$ power-law spectrum, the measured differential flux of astrophysical neutrinos is $E^{2} F(E)=2.9 \times 10^{-8} \mathrm{GeV}^{-2} \mathrm{~s}^{-1} \mathrm{sr}^{-1}$ for the sum of the three evenly distributed neutrino flavors. The sources of observed neutrinos are not yet identified. The detected 37 events are scattered over the sky and do not show evident correlation with any astronomical objects [3]. It seems that Galactic sources might account only for a minority of events. The detected astrophysical neutrinos could be produced in extragalactic sources of ultra high energy protons and nuclei. The discussion about potential sources of very high energy neutrinos in the light of the last experimental results can be found in $[4,5,6]$ where other useful references are given.

The rare extragalactic Type IIn supernova remnants are considered in the present paper as sources of diffuse high energy neutrinos. It is well established that supernova remnants are efficient accelerators of protons, nuclei and electrons. They are the principle sources of Galactic cosmic rays. The diffusive shock acceleration mechanism suggested in $[7,8,9,10]$ can provide the acceleration of protons and nuclei in the most frequent Type IIP, Ia, Ib/c supernova events up to about $10^{15} \mathrm{Z} \mathrm{eV}$ that allows to explain the spectrum and composition of Galactic cosmic rays with a proton-helium knee at $3 \times 10^{15} \mathrm{eV}$ and the maximum energy $\sim 10^{17} \mathrm{eV}$ where iron nuclei dominate, see [11]. Two orders of magnitude larger $E_{\max } \sim 10^{17} \mathrm{eV} /$ nucleon is needed to explain the IceCube neutrino observations. It can be achieved with the Type IIn supernovae that stand out because of extremely dense wind of their progenitor stars with a mass loss rate $10^{-3}-10^{-1} M_{\odot}$ $\mathrm{yr}^{-1}$ [12]. As it will be shown below, the large kinetic energy of explosion and very high gas density in the acceleration region lead to the needed energy of accelerated particles and efficiency of neutrino production in $p p$ interactions.

Diffusive shock acceleration by supernova shocks propagating in dense stellar winds was already considered in $[13,14,15]$. Simple analytical estimates showed that radiowaves, gamma-rays and neutrinos might be observable from the nearest Type IIn supernova remnants if the efficient diffusive shock acceleration takes place in these objects. In the present paper we develop this idea further and investigate whether the flux of neutrinos produced in extragalactic Type IIn supernova remnants can explain the IceCube data. For this purpose we perform numerical modeling of particle acceleration in a supernova remnant produced by Type IIn supernova explosion and calculate neutrino production. Our model of nonlinear diffusive shock acceleration describes the remnant evolution and the production of energetic particles. The detailed description of the model was presented in [16] and the simplified version of the model was used in [11] for the explanation of energy spectrum and composition of Galactic cosmic rays. Similar numerical models of diffusive shock acceleration in supernova remnants were developed and employed in $[17,18,19]$.

The paper is organized as follows. In the next Section 2 we describe modeling of particle 
acceleration and calculate the spectrum of neutrinos produced in Type IIn supernova remnants. These results are used in Section 3 for calculation of diffuse neutrino background in the expanding Universe. The discussion of results and conclusions are given in Sections 4 and 5.

\section{Modeling of diffusive shock acceleration in the remnant of Type IIn supernova}

Details of our model of nonlinear diffusive shock acceleration can be found in [16]. The model contains coupled spherically symmetric hydrodynamic equations and the transport equations for energetic protons, ions and electrons. The forward and reverse shocks are included in the consideration.

The blast wave produced by Type IIn supernova explosion propagates through the wind of the presupernova star. We assume that the initial stellar wind density profile is described by the following expression:

$$
\rho=\frac{\dot{M}}{4 \pi u_{w} r^{2}} .
$$

Here $\dot{M}$ is the mass-loss rate of the supernova progenitor star, $u_{w}$ is the wind velocity and $r$ is the distance from the center of explosion.

We use the following parameters of the supernova explosion. The explosion energy $E_{S N}=10^{52}$ erg, ejecta mass $M_{e j}=10 M_{\odot}, \dot{M}=10^{-2} M_{\odot} \mathrm{yr}^{-1}$, the parameter of ejecta velocity distribution $k=9$ (this parameter describes the power-law density profile $\rho_{e j} \propto r^{-k}$ of the outer part of the ejecta that freely expands after supernova explosion), the stellar wind velocity $u_{w}=100 \mathrm{~km} \mathrm{~s}^{-1}$ that are the characteristic values for Type IIn supernova [12].

The injection efficiency of thermal protons $\eta$ is taken to be independent of time $\eta=0.01$. Protons of mass $m$ are injected at the forward and reverse shocks. The high injection efficiency results in the significant shock modification already at early stage of the supernova remnant expansion.

The secondary electrons and positrons are effectively produced in the dense medium of Type IIn supernova remnant via $p p$ interactions. That is why we do not take into account injection of thermal electrons at the shocks in the present calculations.

Cosmic ray diffusion is determined by particle scattering on magnetic inhomogeneities. The cosmic ray streaming instability increases the level of magnetohydrodynamic turbulence in the shock vicinity [8] and even significantly amplify the absolute value of magnetic field in young supernova remnants $[20,21]$. It decreases the diffusion coefficient and increases the maximum energy of accelerated particles. The results of continuing theoretical study of this effect can be found in review papers $[22,23]$.

In our calculations below, we use the Bohm value of the diffusion coefficient $D_{B}=p v c / 3 q B$, where $q$ is the electric charge of particles. The amplified upstream magnetic field is $B=V_{f} \sqrt{4 \pi \rho} / M_{A}$. Here $V_{f}$ is the speed of the forward shock. The value of the parameter $M_{A}=10$ describing the magnetic field amplification is assumed.

Figures (1)-(2) illustrate the results of our numerical calculations.

We stop our calculations at $t=30 \mathrm{yr}$ when the stellar wind mass swept up by the forward shock is of the order of $20 M_{\odot}$. This value of the total mass loss is expected if the initial mass of the Type IIn supernova progenitor is $60-70 M_{\odot}$. The rest goes into ejecta $\left(\sim 10 M_{\odot}\right)$ and the 


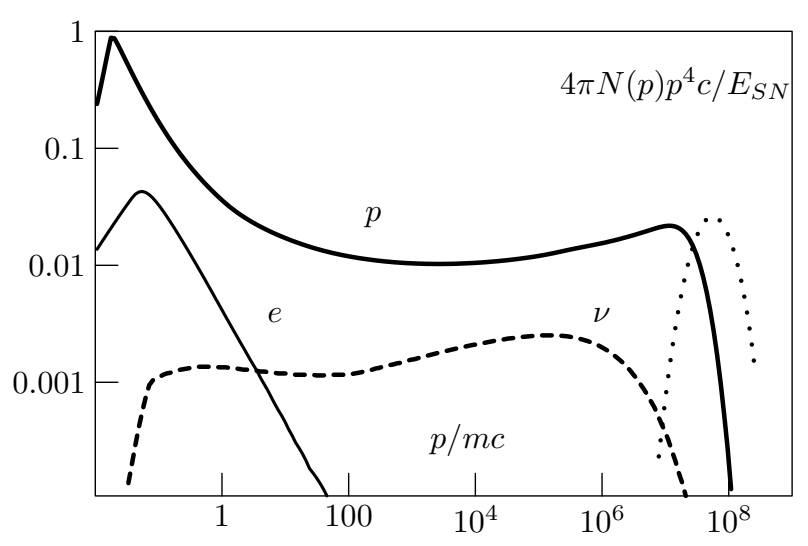

Figure 1: Spectra of particles produced in the supernova remnant during $30 \mathrm{yr}$ after explosion. Spectrum of protons (thick solid line ), spectrum of secondary electrons (multiplied on $10^{3}$, thin solid line), spectrum of neutrinos (thick dashed line) are shown.

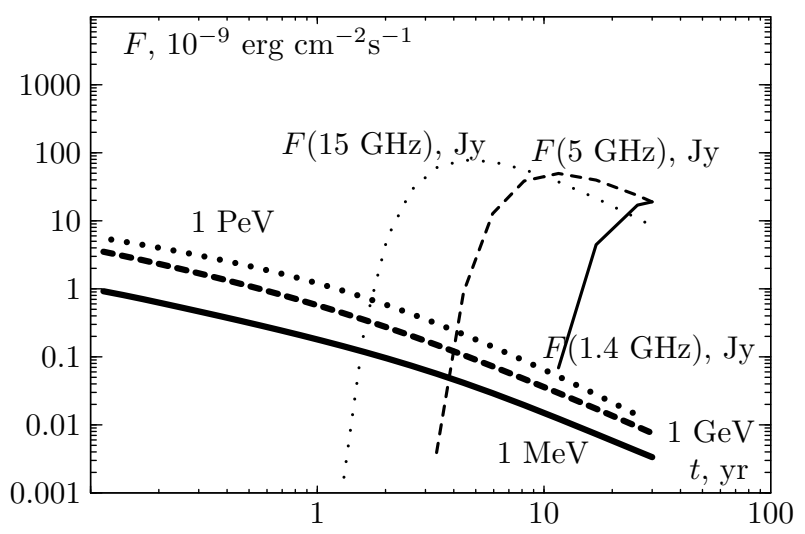

Figure 2: Dependencies on time of fluxes from supernova remnant at distance $1 \mathrm{Mpc}$. We show the neutrino flux at $1 \mathrm{PeV}$ produced via pion decay (thick dotted line), gamma-ray flux at $1 \mathrm{GeV}$ produced via pion decay (thick dashed line). The evolution of synchrotron gamma-ray flux at $1 \mathrm{MeV}$ (thick solid line) and radio-fluxes at $1.4 \mathrm{GHz}, 5 \mathrm{GHz}$ and $15 \mathrm{GHz}$ (thin solid, dashed and dotted lines respectively) are also shown.

black hole $\left(\sim 40 M_{\odot}\right)$. At later times the forward shock enters rarefied medium created by the fast tenuous wind of supernova progenitor at the main sequence stage. So the production of neutrinos becomes negligible at this time.

The spectra of particles produced during 30 years after explosion are shown in Fig.1. They are calculated as the sum of the spectra integrated throughout simulation domain and of the timeintegrated outward diffusive flux at the simulation boundary at $r=2 R_{f}$ where $R_{f}$ is the forward shock radius. The maximum energy of accelerated protons is about $30 \mathrm{PeV}$, while higher energy particles have already left the remnant. We also show the time-integrated spectrum of neutrinos produced in $p p$ interactions. We checked that $p \gamma$ interactions result in the negligible contribution to neutrino fluxes for realistic supernova optical luminosity.

About $25 \%$ of the kinetic energy of explosion goes to cosmic rays. 
Evolution of non-thermal emission produced in the supernova remnant at distance $1 \mathrm{Mpc}$ is shown in Fig.2. We take into account the synchrotron self-absorption and free-free thermal absorption that are important for radio-supernovae [24]. We use the temperature $T=10^{4} \mathrm{~K}$ of circumstellar wind that is a characteristic value for dense stellar winds ionized by radiation coming from the forward and reverse shocks [25].

It is instructive to compare results of the present calculations with the approximate analytical expression for the maximum energy of accelerated protons derived in our paper [11]. The maximum energy of protons $E_{\max }$ accelerated by a supernova shock in the stellar wind was estimated as

$$
E_{\max }=8 \mathrm{PeV}\left(\frac{\dot{M}}{10^{-4} M_{\odot} \mathrm{yr}^{-1}}\right)^{1 / 2}\left(\frac{u_{w}}{100 \mathrm{~km} \mathrm{~s}^{-1}}\right)^{-1 / 2}\left(\frac{E_{S N}}{10^{52} \mathrm{erg}}\right)\left(\frac{M_{e j}}{10 M_{\odot}}\right)^{-1}
$$

A similar estimate for Type Ia supernova explosion in the uniform medium gives the "knee" energy $\sim 3 \mathrm{PeV}$ for protons. The shocks propagating in stellar winds accelerate particles to higher energies. In particular Type IIn supernova remnants with their dense winds with mass-loss rate $\dot{M} \sim$ $10^{-2} M_{\odot} \mathrm{yr}^{-1}$ can accelerate protons up to $80 \mathrm{PeV}$ according to Eq. (2.2). This order of magnitude estimate is in agreement with our numerical results illustrated in Fig. 1. Thus, explanation of proton acceleration up to $\sim 10^{17} \mathrm{eV}$ in Type IIn supernova remnants is in line with the acceleration of cosmic rays up to the knee in Type Ia supernova remnants. The "knee" energy of accelerated particles is reached at the beginning of the Sedov stage when a significant fraction of explosion energy transfers to the shock wave. In denser wind, the amplified magnetic field is stronger that leads to larger $E_{\max }$.

\section{Calculation of background neutrino flux}

The neutrino spectrum produced by a single supernova remnant $Q\left(E_{v}\right)$ (see Fig.1) can be used for the determination of extragalactic neutrino background. Distributed in the Universe Type IIn supernova remnants give the following diffuse flux of neutrinos:

$F\left(E_{v}\right)=\frac{c}{4 \pi H_{0}} \int_{0}^{z_{\max }} d z \frac{Q\left((1+z) E_{v}\right) v_{s n}(1+z)^{m}}{\sqrt{\Omega_{m}(1+z)^{3}+\Omega_{\Lambda}}}=\frac{c}{4 \pi H_{0}} \int_{E_{v}}^{\left(1+z_{\max }\right) E_{v}} d E^{\prime} \frac{E^{\prime m}}{E_{v}^{m+1}} \frac{v_{s n} Q\left(E^{\prime}\right)}{\sqrt{\Omega_{m} E^{\prime 3} / E_{v}^{3}+\Omega_{\Lambda}}}$.

Here the adiabatic energy loss of neutrinos produced at the redshifts $0 \leq z \leq z_{\max }$ is taken into account. The present neutrino production rate per unit energy and volume is $v_{s n} Q\left(E_{v}\right)$, where $v_{s n}$ is the rate of Type IIn supernovae at $z=0$ while the cosmological evolution of the sources in the comoving volume is described as $(1+z)^{m}$ ( $m=0$ implies no evolution). The evolution parameter $m=3.3$ for $z<1$ and no evolution at $z>1$, the maximum redshift $z_{\max }=5$ and the rate $v_{s n}=10^{-6} \mathrm{Mpc}^{-3} \mathrm{yr}^{-1}$ at $z=0$ are assumed in our calculations (see e.g. [26]). This rate of Type IIn supernovae is 100 times lower than the rate of all core collapse supernovae. $H_{0}=70 \mathrm{~km}$ $\mathrm{s}^{-1} \mathrm{Mpc}^{-1}$ is the Hubble parameter at the present epoch, the matter density in the flat Universe is $\Omega_{m}=0.28$, and the $\Lambda$-term is $\Omega_{\Lambda}=0.72$. 




Figure 3: Calculated spectra of neutrino produced by IIn SNRs in the expanding Universe (solid line). IceCube data [2] are also shown.

The calculated background neutrino spectrum is shown in Fig.3. The figure demonstrates very good fit of our calculations to the IceCube data.

Using the same approach we can calculate the flux of extragalactic protons. Our results are compared with cosmic ray data in Fig.4. So the proton flux produced by extragalactic IIn supernova is below the measured cosmic ray fluxes.

The possible contribution of Galactic Type IIn SN events to the observed cosmic ray intensity might be comparable with the Type IIb supernovae as it was calculated in our paper [11]. Both types of supernovae probably produce approximately the same amount of cosmic rays with almost the same maximum energy of particles but SNIIn generate much more secondary particles and neutrinos. The low expected rate of these Galactic supernovae, about one per 5 thousand years, makes estimates of their contribution to the observed intensity of very high energy Galactic cosmic rays rather uncertain.

Simple order of magnitude estimates can be done to clarify how the obtained neutrino flux depends on supernova parameters. The main production of high energy particles and neutrinos is finished at the beginning of the Sedov stage when the shock radius $R_{S}$ can be determined from the condition $M_{e j}=4 \pi \int^{R_{S}} d r r^{2} \rho=\dot{M} R_{S} / u_{w}$. The spectrum $E^{-2}$ is assumed for the accelerated particles. The neutrino energy flux expected from a supernova at distance $D$ can be estimated as (see also $[14,15])$

$$
\begin{gathered}
f\left(E_{v}\right) E_{v}^{2}=\frac{3 \xi_{C R} K_{v}}{16 \pi^{2} \ln \left(E_{\mathrm{max}} / m c^{2}\right)} \frac{c V_{f}^{2} \sigma_{p p} \dot{M}^{2}}{m u_{w}^{2} R_{f} D^{2}}= \\
10^{-8} \mathrm{erg} \mathrm{cm}^{-2} \mathrm{~s}^{-1} \mathrm{t}_{\mathrm{yr}}^{-1} \mathrm{D}_{\mathrm{Mpc}}^{-2} \xi_{\mathrm{CR}}\left(\frac{\dot{\mathrm{M}}}{10^{-2} \mathrm{M}_{\odot} \mathrm{yr}^{-1}}\right)^{2}\left(\frac{\mathrm{u}_{\mathrm{w}}}{100 \mathrm{~km} \mathrm{~s}^{-1}}\right)^{-2}\left(\frac{\mathrm{E}_{\mathrm{SN}}}{10^{52} \mathrm{erg}}\right)^{1 / 2}\left(\frac{\mathrm{M}_{\mathrm{ej}}}{10 \mathrm{M}_{\odot}}\right)^{-1 / 2}
\end{gathered}
$$

Here $\xi_{C R}$ is the ratio of cosmic ray pressure to the ram pressure of the shock $\rho V_{f}^{2}, K_{V} \approx 0.25$ is the fraction of energy that goes into neutrinos in $p p$ interactions, $\sigma_{p p}$ is the cross-section of $p p$ interactions and $E_{\max }$ is the maximum energy of accelerated protons given by Eq. (2.2). The 
expression is valid when the shock radius $R_{f}>R_{\min }=c \sigma_{p p} \dot{M} / 4 \pi u_{w} m V_{f}$ and the energy losses via $p p$ interactions are smaller than the adiabatic losses.

Expression (3.2) can be used for the determination of neutrino background produced by all Type IIn supernovae:

$$
\begin{gathered}
F\left(E_{v}\right) E_{v}^{2}=\frac{3 \xi_{C R} K_{v}}{16 \pi^{2} \ln \left(E_{\max } / m c^{2}\right)} \frac{v_{s n} c^{2} V_{f} \sigma_{p p} \dot{M}^{2}}{H_{0} m u_{w}^{2}} \ln \frac{R_{S}}{R_{\min }}=10^{-11} \mathrm{erg} \mathrm{cm}^{-2} \mathrm{~s}^{-1} \mathrm{sr}^{-1} \times \\
\xi_{C R}\left(\frac{\dot{M}}{10^{-2} M_{\odot} \mathrm{yr}^{-1}}\right)^{2}\left(\frac{M_{e j}}{10 M_{\odot}}\right)^{-1 / 2}\left(\frac{u_{w}}{100 \mathrm{~km} \mathrm{~s}^{-1}}\right)^{-2}\left(\frac{E_{S N}}{10^{52} \mathrm{erg}}\right)^{1 / 2}\left(\frac{v_{s n}}{10^{-6} \mathrm{Mpc}^{-3} \mathrm{yr}^{-1}}\right),
\end{gathered}
$$

The background flux is higher if the cosmological evolution of supernova rate is taken into account (see Eq. (3.1)). These estimates are valid at neutrino energies $E_{v}<0.05 E_{\max }$. They confirm the efficiency of high energy neutrino production in supernova explosion in very dense wind of a progenitor star, as it takes place in Type IIn supernovae.

\section{Discussion}

Our calculations show that the IceCube data can be explained by the neutrinos from Type IIn supernovae. If so, the arrival direction of every IceCube neutrino should coincide with the direction to some Type IIn supernova. However, we observe Type IIn supernovae with the redshifts $z<0.1$ while the background is determined by the supernovae with $z \sim 1$. Therefore we expect that only several percent of observed neutrinos i.e. about one IceCube neutrino event are associated with the known Type IIn supernovae. Unfortunately, the arrival directions of majority of IceCube neutrinos are known with low angular resolution (about 15 degrees). We found that several Type IIn supernovae were indeed observed in the vicinity of some IceCube neutrinos. However this result has low statistical significance.

Nevertheless we note that the arrival direction of one IceCube PeV neutrino candidate (event 20 ) is within 5 angular degrees from the SN 1978K. This nearest IIn supernova in the galaxy NGC 1313 at distance $4 \mathrm{Mpc}$ is for decades observed in radio, X-rays and optics (e.g. [28]). The massloss rate of SN $1978 \mathrm{~K}$ progenitor is estimated as $\dot{M}=2 \cdot 10^{-3} M_{\odot} \mathrm{yr}^{-1}$ [29] that is 5 times lower than we use in our calculations. Thus the fluxes expected from this supernova are not so high as ones shown in Fig.2. In spite of this it is possible that the $\mathrm{PeV}$ neutrino candidate was emitted by SN 1978K.

The arrival direction of another IceCube PeV neutrino candidate (event 35) is within 10 angular degrees from the SN 1996cr. This nearest IIn supernova in the Circinus galaxy at distance 4 Mpc was also detected in radio, X-rays and optics (e.g. [30]). The Circinus galaxy is a bright source of $\mathrm{GeV}$ gamma rays [31]. It is known that the forward shock of SN1996cr entered the dense shell of circumstellar matter. The radio flux of this supernova is only several times lower than the flux shown in Fig.2. That is why this IceCube PeV neutrino candidate could be emitted by SN 1996cr.

On the other hand, 8 of 37 IceCube neutrinos left tracks. The arrival directions of these neutrinos are determined with good angular resolution (about 1 degree). We found that none of these neutrinos is associated with known Type IIn supernovae. The expected number of coincidences is of the order of 0.3. Thus, only one track neutrino can be observed from the vicinity of Type IIn supernova during one decade of IceCube operation. 


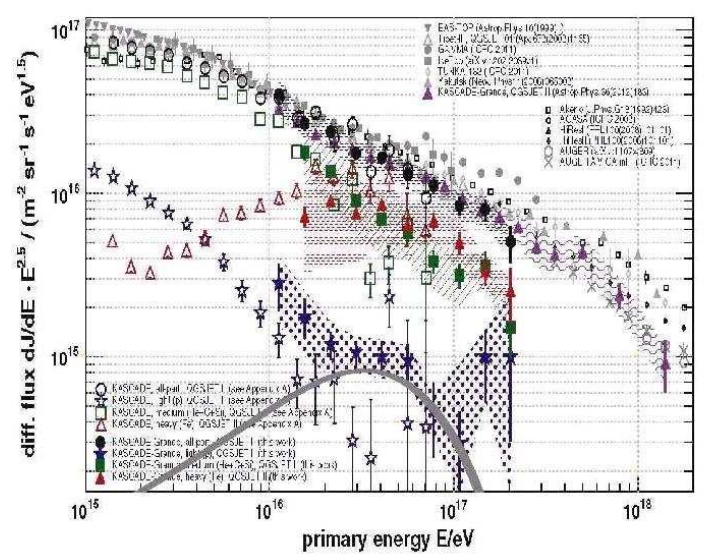

Figure 4: Comparison of calculated spectrum of cosmic ray protons produced by extragalactic IIn SNRs in the expanding Universe (gray solid line) and available cosmic ray data [27].

\section{Conclusions}

Our main conclusions are the following:

1) The diffusive shock acceleration of particles in Type IIn supernova remnants and the production of neutrinos via $p p$ interactions in the dense presupernova winds can explain the diffuse flux of high energy astrophysical neutrinos observed in the IceCube experiment.

2) The calculated maximum energy of protons accelerated in remnants of IIn supernova is close to $10^{17} \mathrm{eV}$. This value is higher than the maximum energy achieved in the main part of SNRs and is explained by the high density of the circumstellar matter.

3) The efficient acceleration of particles and production of secondary electrons and positrons result in the fluxes of radiowaves and gamma-rays that can be observed from the nearest Type IIn supernova remnants.

4) Future IceCube operation and the search of correlations between neutrino arrival directions and the directions to Type IIn supernovae may check whether these objects are the sources of high energy neutrinos but only one track event during 10 years is expected from these supernovae with the redshifts less than 0.1. The bright phase of a Type IIn supernova remnant as a source of PeV neutrinos lasts for about several years after the supernova explosion.

The work was supported by the Russian Foundation for Basic Research grant 13-02-00056 and by the Russian Federation Ministry of Science and Education contract 14.518.11.7046.

\section{References}

[1] Aartsen, M.G., Ackermann, M., Adams, J. et al. 2013, Science 342, 1242856

[2] Aartsen, M.G., Ackermann, M., Adams, J. et al. 2014, Phys. Rev. Lett., 113, 1101

[3] Aartsen, M.G., Ackermann, M., Adams, J. et al. 2014, ApJ, 796, 109

[4] Anchordoqui, L.A., Barger, V., Cholis, E. et al. 2014, JHEAp, 1, 1

[5] Gaisser, T. \& Halzen F. 2014, Annu. Rev. Nucl. Part. Sci. 64, 101 
[6] Murase, K 2014, arXiv:1410.3680v2

[7] Krymsky, G.F. 1977, Soviet Physics-Doklady, 22, 327

[8] Bell, A.R., 1978, MNRAS, 182, 147

[9] Axford, W.I., Leer, E. \& Skadron, G., 1977, Proc. 15th Int. Cosmic Ray Conf., Plovdiv, 90, 937

[10] Blandford, R.D., \& Ostriker, J.P. 1978, ApJ, 221, L29

[11] Ptuskin, V.S., Zirakashvili, V.N. \& Seo, E.S. 2010, ApJ, 718, 31

[12] Moriya, T.J., Maeda, K., Taddia, F., Sollerman, J., Blinnikov, S.I., \& Sorokina, E.I., 2014, MNRAS 439, 2917

[13] Murase, K., Thompson, T.A., Lacki, B.C., \& Beacom, J.F., 2011, Phys. Rev. D, 84, 043003

[14] Kats, B., Sapir, N., \& Waxman, E., 2011, arXiv.1106.1898

[15] Murase, K., Thompson, T.A., \& Ofek, E.O., 2014, MNRAS 440, 2528

[16] Zirakashvili, V.N. \& Ptuskin V.S. 2012, APh 39, 12

[17] Berezhko, E.G., Elshin, V.K. \& Ksenofontov, L.T., 1994, APh 2, 215

[18] Kang, H. \& Jones, T.W. 2006, APh 25, 246

[19] Berezhko, E.G., \& Völk, H.J., 2007, ApJ, 661, L175

[20] Bell, A.R., 2004, MNRAS, 353, 550

[21] Zirakashvili, V.N. \& Ptuskin, V.S., 2008, ApJ 678, 939

[22] Bell, A.R., 2014, APh 43, 56

[23] Caprioli, D., 2014, arXiv:1410.1644v1

[24] Chevalier, R., 1998, ApJ 499, 810

[25] Lundqvist, P., \& Fransson, C., 1988, A\&A 192, 221

[26] Dahlen, T., Strolger, L.-G., Riess, A.G. et al., 2012, ApJ, 757, 70

[27] Apel, W.D., Arteaga-Velázquez, J.C., Bekk, K. et al. 2013, APh 47, 54

[28] Smith, I.A., Ryder, S.D., Böttcher, M., et al., 2007, ApJ, 669, 1130

[29] Chugai, N.N., Danziger, I.J., \& Della Valle, M., 1995, MNRAS, 276, 530

[30] Bauer, F.E., Dwarkadas, V.V., Brandt, W.N. et al., 2008, ApJ, 688, 1210

[31] Hayashida, M., Stawarz, L., Cheung, C.C., et al. 2013, ApJ, 779, 131 